\title{
The influence of heat capacity assumptions on the estimation of solubility parameters from solubility data
}

\author{
Steven H. Neau ${ }^{1}$, Gordon L. Flynn ${ }^{1}$ and Samuel H. Yalkowsky ${ }^{2}$ \\ ${ }^{I}$ College of Pharmacy, University of Michigan, Ann Arbor, MI 48109.1065 (U.S.A.) \\ and ${ }^{2}$ College of Pharmacy, the University of Arizona, Tucson, AZ 85721 (U.S.A.)
}

(Received 25 February 1988)

(Modified version received 8 June 1988)

(Accepted 13 July 1988)

\section{Summary}

Regular solution theory indicates that solubility parameters of crystalline organic compounds can be estimated from solubilities in London solvents. The equation for this purpose is:

$\ln X_{2}=-\left\{\frac{\Delta \bar{H}_{\mathrm{f}}}{R T_{\mathrm{f}}}\right\} \frac{\left(T_{\mathrm{f}}-T\right)}{T}+\left\{\frac{\Delta C_{\mathrm{p}}}{R}\right\}\left[\frac{\left(T_{\mathrm{f}}-T\right)}{T}-\ln \left(\frac{T_{\mathrm{f}}}{T}\right)\right]-\left(\frac{\bar{V}_{2} \phi_{1}^{2}}{R T}\right)\left(\delta_{1}-\delta_{2}\right)^{2}$

where $X_{2}$ is the mole fraction solubility of a compound in a solvent with a solubility parameter of $\delta_{1}$. With the exception of $\Delta C_{\mathrm{p}}$, all parameters in the equation necessary to estimate the solute solubility parameter, $\delta_{2}$, can either be suitably approximated or readily determined experimentally. In order to use the equation, simplifying assumptions have been made concerning $\Delta C_{\mathrm{p}}$, namely: $\Delta C_{\mathrm{p}}=0$ or $\Delta C_{\mathrm{p}}=\Delta S_{\mathrm{f}}$, the entropy of fusion. In the present work, we have considered the extent to which these assumptions influence the magnitude of solubility parameters estimated from solubilities in $n$-hexane, $n$-heptane, $n$-dodecane, cyclohexane, carbon tetrachloride, toluene and benzene. Using $n$-alkyl $p$-aminobenzoates as test compounds, it is shown that solubility-based solubility parameters are relatively insensitive to the form of the equation used to calculate $\delta_{2}$. Specifically, solubility parameter estimations based on the two simplifying assumptions differ by no more than $0.2(\mathrm{cal} / \mathrm{ml})^{1 / 2}$, an increment of the order of the presumed inherent error of estimation.

\section{Introduction}

Much work has been published on the determination of solubility parameters from solution data (Cove et al., 1980; Chertkoff and Martin, 1960; Hagen and Flynn, 1983; Hildebrand et al., 1970; James and Roberts, 1968; Khalil et al., 1976; Martin et al., 1980; Martin and Carstensen, 1981; Restaino and Martin, 1964; Sunwoo and Eisen, 1971). Such determinations are usually

Correspondence: G.L. Flynn, College of Pharmacy, University of Michigan, Ann Arbor, MI 48109-1065, U.S.A. based on the use of the Hildebrand-Scatchard equation in its familiar form:

$\ln X_{2}=\ln a_{2}-\frac{\overline{V_{2}} \phi_{1}^{2}}{R T}\left(\delta_{1}-\delta_{2}\right)^{2}$

where $R$ is the gas constant, $T$ is the experimental temperature, $X_{2}$ is the mole fraction solubility of the solute, $a_{2}$ is the ideal activity of the solute, $V_{2}$ is the molar volume of the liquid solute, $\phi_{1}$ is the volume fraction of the solvent and $\delta_{1}$ and $\delta_{2}$ are the solubility parameters of the solvent and solute, respectively ${ }^{4}$. The latter were defined by Hildebrand and Scott (1950) as being equal to the 
square-root of the cohesive energy density of the pure liquid form of the solvent and solute, respectively:

$\delta_{\mathrm{i}}=c_{\mathrm{ii}}^{1 / 2}$

The units for $\delta_{i}$ are $(\mathrm{cal} / \mathrm{ml})^{1 / 2}$, now designated a hildebrand.

For a solute which is a solid at the experimental temperature, the ideal solubility is related to the energy required to convert that solid to the super-cooled liquid and is given by:

$$
\begin{aligned}
\ln a_{2}= & -\left(\frac{\Delta \bar{H}_{\mathrm{f}}}{R T_{\mathrm{f}}}\right)\left(\frac{T_{\mathrm{f}}-T}{T}\right)+\left(\frac{\Delta C_{\mathrm{p}}}{R}\right)\left[\left(\frac{T_{\mathrm{f}}-T}{T}\right)\right. \\
& \left.-\ln \left(\frac{T_{\mathrm{f}}}{T}\right)\right]
\end{aligned}
$$

where $\Delta H_{\mathrm{f}}$ is the enthalpy of fusion and $T_{\mathrm{f}}$ is the melting point in degrees Kelvin. $\Delta C_{\mathrm{p}}$ is the difference in heat capacity at constant pressure between the solid form and the hypothetical supercooled liquid form of the compound, both at the same temperature. Since the heat capacity of the super-cooled liquid normally cannot be determined, a means of assigning a fair value to $\Delta C_{\mathrm{p}}$ has been sought. According to one line of reasoning, $\Delta C_{\mathrm{p}}$ is small and can be assumed to be zero (Hagen and Flynn, 1983; Yalkowsky et al., 1972). Another common assumption is that $\Delta C_{\mathrm{p}}$ is equal to the entropy of fusion ${ }^{4,8}, \Delta S_{\mathrm{f}}$, where:

$\Delta \bar{S}_{\mathrm{f}}=\frac{\Delta \bar{H}_{\mathrm{f}}}{T_{\mathrm{f}}}$

The influence of these two assumptions on the value of the solubility parameter, as determined by analyzing solubility data using Regular Solution Theory, will be examined here.

Regular Solution Theory represents the total deviation from ideality as resting in an excess enthalpy of mixing in going from an ideal solution to a regular solution. It is assumed that a regular solution has no excess entropy of mixing (Hildebrand, 1929) with the entropy of mixing being equal to the statistical mixing found in an ideal solution:

$\Delta \bar{S}_{2, \operatorname{mix}}=-R \ln X_{2}$

This has proved to be a valid assumption whenever there is no excess volume of mixing.

Flory and Huggins developed a theory to estimate the entropy of mixing for systems where the solute and solvent are of very different molecular size (Kertes, 1965). The equation they derived:

$\Delta \bar{S}_{2, \text { mix }}=\ln \phi_{2}-\phi_{1}\left(1-\frac{\bar{V}_{2}}{\bar{V}_{1}}\right)$

was applied to the data for this study but the difference between the two estimates of the entropy of mixing was not significant due to the similarity in size between the solutes and the solvents. Eqn. 5, therefore, was employed.

In creating a mixture of a liquid solute in a solvent, it is necessary to overcome the respective cohesive energies that condense the pure liquid solute, $V_{2} c_{22}$, and the equivalent volume of the pure liquid solvent, $V_{2} c_{11}$. There is a return of energy from the cohesive energy between the two components, $V_{2} c_{12}$. Scatchard (1931) assumed that the cohesive energy density of a binary mixture could be estimated from the geometric mean of the cohesive energy densities of the pure liquid components:

$c_{12}=\left(c_{11} c_{22}\right)^{1 / 2}$

This appears to be a good approximation only for non-polar systems where intermolecular forces are primarily London (dispersion) forces. For systems where the components have very different shapes or where they may interact when mixed to form complexes between like or unlike molecules, this is almost certainly not an appropriate assumption (Hildebrand et al., 1970). In the systems studied here, the solutes and solvents are similar in molar volume, and the solute, in most instances, is sufficiently dilute that intermolecular associations in the solution are limited to non-orienting London and Debye (dipole-induced dipole) forces. Thus, 
from the solution standpoint, the geometric mean assumption should be valid. The solvents chosen are incapable of interacting by other than dispersion forces (induced dipoles). It is recognized that interactions in the pure molten solute (supercooled liquid) in this study include strong, orienting hydrogen bonds and Keesom (dipolar) forces. Apparently, the entropy increase associated with the disruption of the order in the solute melt is small within the total thermodynamic scheme, such that this causes no observable departure from regular solution theory. The geometric mean assumption does not seem to be invalidated. The exchange energy density (Hildebrand et al., 1970) then becomes:

$A_{12}=c_{11}+c_{22}-2\left(c_{11} c_{22}\right)^{1 / 2}$

Introducing the solubility parameters, the squareroots of the cohesive energy densities yield:

$A_{12}=\delta_{1}^{2}+\delta_{2}^{2}-2\left(\delta_{1} \delta_{2}\right)=\left(\delta_{1}-\delta_{2}\right)^{2}$

giving the right-hand term in Eqn. 1 its form:

$\ln X_{2}-\ln a_{2}=-\left(\frac{\bar{V}_{2} \phi_{1}^{2}}{R T}\right)\left(\delta_{1}-\delta_{2}\right)^{2}$

In this study, the solubilities of non-electrolyte solutes in non-polar organic solvents are considered with respect to these equations. The solutes, though they possess permanent dipoles, should not experience solute-solute interactions if highly dilute. On this basis, the saturated solutions of these solutes were considered to represent regular solutions as, through the choice of solvents, the interactions between solvent and solute are essentially limited to London and Debye forces.

\section{Materials and Methods}

\section{Chemicals}

All solvents were reagent grade and included $n$-hexane (Fisher), $n$-heptane (Columbus Chem. Ind.), n-dodecane, cyclohexane (Sigma), carbon tetrachloride (Mallinckrodt), toluene (Fisher) and benzene (Baker). Each solvent was used as received. HPLC-grade methanol (Mallinckrodt) and distilled, deionized water were used to dilute samples in preparation for their UV analysis.

The chemicals used as solutes were esters of $p$-aminobenzoic acid; all are solids at room temperature. The first 4 esters in this series, the methyl (Aldrich; Eastman), ethyl (Sigma), $n$-propyl (ICN $\mathrm{K} \& \mathrm{~K}$ ) and $n$-butyl (Sigma) esters, were commercially available. The $n$-pentyl ester was synthesized according to a literature procedure (Flynn and Yalkowsky, 1972) in which the $p$-nitrobenzoate was prepared first and then hydrogenated to the $p$-aminobenzoate.

The pentyl ester of $p$-nitrobenzoic acid was synthesized by refluxing at least a $10 \%$ excess of $p$-nitrobenzoyl chloride (Aldrich) with $n$-pentanol (Sigma) in chloroform (Fisher) for several hours. The chloroform solution was washed with $10 \%$ sodium carbonate (Baker) until free from $p$ nitrobenzoic acid. The chloroform solution was then washed twice with water, dried with magnesium sulfate and the chloroform was evaporated. The nitro group was reduced using a $5 \%$ palladium-on-charcoal catalyst (MCB. Manuf. Chem.) and a Parr hydrogenator. The solvent used for the reduction process was absolute ethanol (U.S. Ind. Chem. Co.). The charcoal was filtered off, the solvent was evaporated and the crude $p$-aminobenzoate was recrystallized several times from $n$ hexane (Fisher).

\section{Solubility determinations}

The solubilities of the $p$-aminobenzoates were obtained by equilibrating an excess of solid with the particular solvent in a stoppered, water-jacketed, glass container. The temperature of the solution was maintained at $25^{\circ} \mathrm{C}$ by a constanttemperature water bath. The slurries were vigorously and continuously mixed using magnetic stirring bars. Preliminary work indicated a saturated state was achieved well within one day. Therefore, samples were taken after at least $24 \mathrm{~h}$ of mixing by means of glass wool-tipped volumetric pipets. The solvent from each individual sample was then evaporated. The sample's solute residue was reconstituted in a known volume of HPLC-grade methanol $(25$ or $50 \mathrm{ml})$ and was diluted with 
methanol and distilled, deionized water, as necessary, for assay. Because of the low volatility of $n$-dodecane, samples in this solvent were diluted directly with HPLC-grade methanol prior to assay. The diluted samples were analyzed by UV spectrophotometry, using a Hewlett-Packard $8450 \mathrm{~A}$ or a Perkin-Elmer Lambda 7 UV/VIS Spectrophotometer with the wavelength selected from the absorbance maximum in the range 280-300 $\mathrm{nm}$. In developing the research procedures, considerable effort was put into HPLC analysis of the samples. It was found that direct UV assay of diluted samples gave identical results and therefore the latter was adopted as the procedure.

\section{Differential scanning calorimetry}

The melting points and the enthalpies of fusion of the $p$-aminobenzoates were determined by differential scanning calorimetry. The calorimeter in both cases was calibrated with an indium standard. Accurately weighed samples (1-10 mg) were placed in aluminum crucibles on which an aluminum lid was crimped. An empty aluminum crucible and lid served as the reference. Samples were heated at $1^{\circ} \mathrm{C} / \mathrm{min}$ for melting point determinations and at $1-10^{\circ} \mathrm{C} / \mathrm{min}$ for enthalpy of fusion determinations.

Melting points were taken as the onset temperature of the melting endotherm from a Mettler FP800 Thermosystem. Enthalpies of fusion were determined on a Perkin-Elmer DSC-2C equipped with a Thermal Analysis Data Station, and were calculated by comparison of the area under the curve of a sample endotherm with the area under the curve of the reference compound, indium, which has a known enthalpy of fusion of 6.81 $\mathrm{cal} / \mathrm{g}$.

\section{Results and Discussion}

A "best-fit" solubility parameter was determined for each ester using the solubility of the ester in each of the 7 solvents, the enthalpy of fusion and melting point, and first one, and then the other, of the two $\Delta C_{\mathrm{p}}$ assumptions. Molar volumes were approximated using an additivity
TABLE 1

Atomic and group contributions to the molar volume

\begin{tabular}{lllc}
\hline Atom & $\Delta Y_{\mathrm{i}}(\mathrm{ml} / \mathrm{mol})$ & Group & $\Delta V_{\mathrm{i}}(\mathrm{ml} / \mathrm{mol})$ \\
\hline $\mathrm{C}$ & 9.9 & $-\mathrm{CH}_{3}$ & 32.7 \\
$\mathrm{H}$ & 3.1 & $-\mathrm{CH}_{2}-$ & 16.2 \\
& & $-\mathrm{NH}_{2}$ & 7.7 \\
& & $-\mathrm{COO}_{-}$ & 15.9 \\
& & Ring & -8.1 \\
\hline
\end{tabular}

Yalkowsky, S.H. and Zografi, G., Calculation of partial molal volume in micellar systems. J. Pharm. Sci., 61 (1972) 793-795.

\begin{tabular}{llll}
\hline Ester & $\sum \Delta V_{\mathrm{i}}(\mathrm{ml} / \mathrm{mol})$ & Ester & $\sum \Delta V_{\mathrm{i}}(\mathrm{ml} / \mathrm{mol})$ \\
\hline Methyl & 120.0 & $n$-Butyl & 168.6 \\
Ethyl & 136.2 & $n$-Pentyl & 184.8 \\
$n$-Propyl & 152.4 & & \\
\hline
\end{tabular}

method (Yalkowsky and Zografi, 1972) and are given in Table 1. The value of $\phi_{1}$ is given by:

$\phi_{1}=\frac{X_{1} \bar{V}_{1}}{\left(X_{1} \bar{V}_{1}+X_{2} \bar{V}_{2}\right)}$

Since the solutions were not consistently dilute, $\phi_{1}$ could not be assumed to be unity. This determination then required the rearrangement of Eqn. 1 to:

$\left(\ln X_{2}-\ln a_{2}\right)\left(\frac{R T}{\bar{V}_{2} \phi_{1}^{2}}\right)=\left(\delta_{1}-\delta_{2}\right)^{2}$

Values of $\ln a_{2}$, the ideal activity of the solute, were determined using Eqn. 3 and one of the $\Delta C_{p}$ assumptions. These are tabulated in Table 2 .

A summary of solubilities of the esters in each of the seven solvents is presented in Table 3.

TABLE 2

Ideal activities of the p-aminobenzoates at $25^{\circ} \mathrm{C}$

\begin{tabular}{lllll}
\hline Ester & $\Delta H_{\mathrm{f}}(\mathrm{cal} / \mathrm{mol})$ & $T_{\mathrm{f}}(\mathrm{K})$ & \multicolumn{1}{l}{$\ln a_{2}$} \\
\cline { 3 - 5 } & & & $\Delta C_{\mathrm{p}}=0$ & $\Delta C_{\mathrm{p}}=\Delta S_{\mathrm{f}}$ \\
\hline Methyl & 5390 & 385 & -2.05 & -1.80 \\
Ethyl & 5320 & 363 & -1.60 & -1.45 \\
$n$-Propyl & 4910 & 347 & -1.16 & -1.08 \\
$n$-Butyl & 4890 & 331 & -0.801 & -0.761 \\
$n$-Pentyl & 5720 & 325 & -0.810 & -0.776 \\
\hline
\end{tabular}


TABLE 3

Experimental solubility data for the p-aminobenzoates

\begin{tabular}{|c|c|c|c|c|c|c|}
\hline \multirow[t]{2}{*}{ Solvent } & \multirow[t]{2}{*}{$\delta_{1}^{*}$} & \multicolumn{5}{|c|}{ Mean $\ln X_{2}$ (S.D.) } \\
\hline & & Methyl & Ethyl & n-Propyl & n-Butyl & $n$-Pentyl \\
\hline$\overline{n-H e x a n e}$ & 7.27 & $-7.93(0.013)$ & $-7.07(0.014)$ & $-6.48(0.003)$ & $-6.03(0.005)$ & $-5.80(0.024)$ \\
\hline$n$-Heptane & 7.50 & $-7.81(0.007)$ & $-6.99(0.019)$ & $-6.42(0.006)$ & $-5.91(0.029)$ & $-5.73(0.014)$ \\
\hline$n$-Dodecane & 7.92 & $-7.34(0.027)$ & $-6.63(0.004)$ & $-6.09(0.023)$ & $-5.58(0.015)$ & $-5.29(0.028)$ \\
\hline Cyclohexane & 8.19 & $-7.77(0.018)$ & $-6.91(0.006)$ & $-6.20(0.008)$ & $-5.60(0.009)$ & $-5.33(0.016)$ \\
\hline Carbon tetrachloride & 8.55 & $-5.77(0.002)$ & $-4.68(0.004)$ & $-3.55(0.001)$ & $-1.50(0.005)$ & $-1.24(0.031)$ \\
\hline Toluene & 8.93 & $-4.22(0.007)$ & $-3.13(0.007)$ & $-2.07(0.004)$ & $-1.20(0.016)$ & $-1.09(0.023)$ \\
\hline Benzene & 9.16 & $-3.94(0.014)$ & $-2.72(0.006)$ & $-1.64(0.004)$ & $-1.12(0.014)$ & $-0.98(0.013)$ \\
\hline
\end{tabular}

* Hoy, K.L., New values of the solubility parameters from vapor pressure data. J. Paint Technol., 42 (1970) 76-118.

Solubility parameters were determined from this data using a Gauss-Newton regression analysis using Eqn. 12. The "best-fit" $\delta_{2}$ values are summarized in Table 4 . It is easily seen and is very significant that the assumption regarding the differential heat capacity has no greater effect on the estimate of solubility parameters than the intrinsic error of the experimental method itself. The assumption does affect the value of the ideal solubility, $\ln a_{2}$, and this quantity determines the maximum for the parabola generated by Eqn. 1, but the position for the maximum on the solubility parameter axis is only marginally shifted (see Fig. 1).

Note that the alkane solvents are on the extreme left-hand side of the curve, with solubility values well-removed from ideal solubility due to the significant difference in the solubility parame-

TABLE 4

Comparison of solubility parameters from experimental results

\begin{tabular}{|c|c|c|c|}
\hline \multirow[t]{3}{*}{ Ester } & \multicolumn{3}{|c|}{$(\mathrm{cal} / \mathrm{ml})^{1 / 2}$} \\
\hline & \multicolumn{2}{|c|}{$\delta_{2}$ (S.D.) (Exptl) ${ }^{a}$} & \multirow{2}{*}{$\frac{\delta_{2}{\text { (Hex })^{b}}^{b}}{\Delta C_{\mathrm{p}}=0}$} \\
\hline & $\Delta C_{\mathrm{p}}=0$ & $\Delta C_{\mathrm{p}}=\Delta S_{\mathrm{f}}$ & \\
\hline Methyl & $12.8(0.2)$ & $13.0(0.2)$ & 12.7 \\
\hline Ethyl & $12.3(0.2)$ & $12.4(0.2)$ & 12.2 \\
\hline n-Propyl & $11.9(0.2)$ & $12.0(0.2)$ & 11.8 \\
\hline n-Butyl & $11.7(0.2)$ & $11.7(0.2)$ & 11.6 \\
\hline$n$-Pentyl & $11.3(0.2)$ & $11.4(0.2)$ & 11.3 \\
\hline
\end{tabular}

a Solubility parameter calculated according to Eqn. 12 with the assumption $\Delta C_{\mathrm{p}}=0$ or $\Delta C_{\mathrm{p}}=\Delta S_{\mathrm{f}}$, the entropy of fusion. b Solubility parameter calculated from hexane solubility only, using Eqn. 12 and the assumption $\Delta C_{\mathrm{p}}=0$. ters of the solute and solvent. On this basis, data from these solvents are likely to be the least influenced by the $\Delta C_{\mathrm{p}}$ assumption. Though some such solvents have been reported to experience a deviation from the geometric mean assumption (Funk and Prausnitz, 1970; Hildebrand et al., 1970), two alkane hydrocarbons used in this study, namely $n$-hexane and $n$-heptane, apparently adhere well to this assumption. Arriving at the solu-

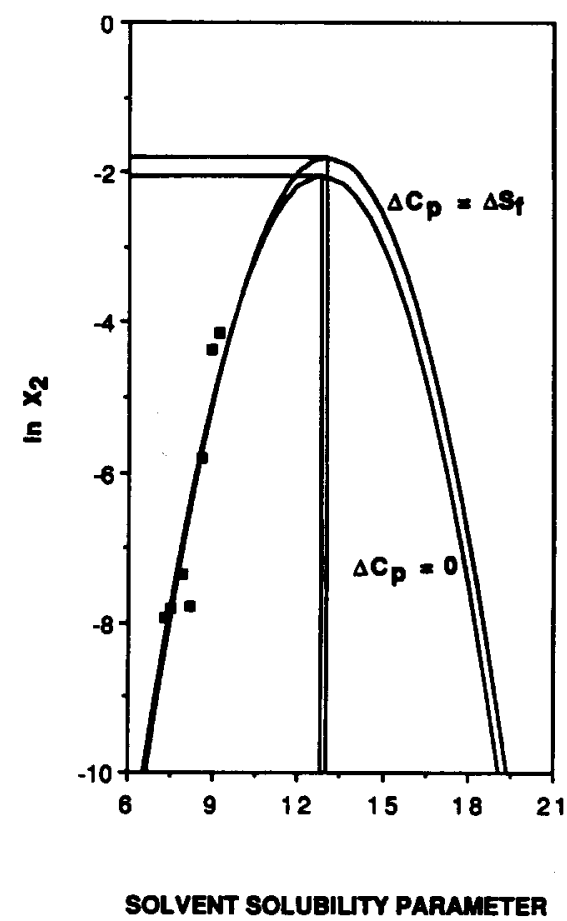

Fig. 1. Methyl ester solubility data. 
bility parameter for an organic compound by a multiple-solvent solubility study is a tedious process. It can be seen from the last column in Table 4 that solubility parameters for these solutes can be more easily arrived at by assessing their solubilities in a single solvent like $n$-hexane or $n$-heptane. One still has to have knowledge of the enthalpy of fusion and the melting point to use Eqns. 1 and 3 for the estimate.

The effect of the $\Delta C_{\mathrm{p}}$ assumption can be shown mathematically. If $\Delta C_{\mathrm{p}}$ is assumed to be zero, Eqn. 1 becomes:

$\ln X_{2}=-\left(\frac{\Delta \bar{S}_{\mathrm{f}}}{R}\right)\left(\frac{T_{\mathrm{f}}-T}{T}\right)-\left(\frac{\bar{V}_{2} \phi_{1}^{2}}{R T}\right)\left(\delta_{1}-\delta_{2}\right)^{2}$

If $\Delta C_{\mathrm{p}}$ is assigned the value of the entropy of fusion, Eqn. 1 becomes:

$$
\ln X_{2}=-\left(\frac{\delta \bar{S}_{\mathrm{f}}}{R}\right)\left(\ln \left(\frac{T_{\mathrm{f}}}{T}\right)\right)-\left(\frac{\bar{V}_{2} \phi_{1}^{2}}{R T}\right)\left(\delta_{1}-\delta_{2}\right)^{2}
$$

The only difference in the two equations is the term involving the entropy of fusion. For a very small difference between the melting point and the solution temperature, the difference between the equations is negligible. In this paper, the largest difference between the melting point and the solution temperature is $87^{\circ} \mathrm{C}$ for the methyl ester. The difference in ideal solubility created by the alternate assumptions for $\Delta C_{\mathrm{p}}$ is $12 \%$, based on the values for $\ln a_{2}$ given in Table 2. This amounts to a difference of $22 \%$ on a mole fraction basis. The influence of this difference can be seen in the 0.2 hildebrand variation in the $\delta_{2}$ values in Table 4 . This is not considered to be a significant difference. The $n$-butyl ester, with its low melting point and relatively low enthalpy of fusion, experiences no difference in the calculated $\delta_{2}$ values, as shown in Table 4 . In this case, the $\Delta C_{\mathrm{p}}$ assumptions make only a $5 \%$ difference in $\ln a_{2}$ as seen in Table 2, which is only a $4 \%$ difference in mole fraction ideal solubility. For the $n$-butyl ester, then, these assumptions had no influence on the
TABLE 5

Physical properties and solubilities of hydrocortisone

\begin{tabular}{llll}
\hline Solvent & $\delta_{1}{ }^{*}$ & $X_{2}$ & $\ln X_{2}$ \\
\hline$n$-Hexane & 7.27 & $1.24 \times 10^{-8}$ & -18.21 \\
Cyclohexane & 8.19 & $2.24 \times 10^{-7}$ & -15.31 \\
Carbon tetrachloride & 8.55 & $2.75 \times 10^{-6}$ & -12.80 \\
Toluene & 8.93 & $2.85 \times 10^{-5}$ & -10.47 \\
Benzene & 9.16 & $3.19 \times 10^{-5}$ & -10.35 \\
\hline
\end{tabular}

* See Table III

$T_{\mathrm{f}}=212^{\circ} \mathrm{C} ; \Delta S_{\mathrm{f}}=16.7 \mathrm{cal} / \mathrm{mol} \mathrm{deg} ; V_{2}=293 \mathrm{ml} / \mathrm{mol}$.

\begin{tabular}{llll}
\hline Assumption & $X_{2, \text { ideal }}$ & $\ln a_{2}$ & $\delta_{2}$ \\
\hline$\Delta C_{\mathrm{p}}=0$ & $5.14 \times 10^{-3}$ & -5.27 & $12.4(0.1)$ \\
$\Delta C_{\mathrm{p}}=\Delta S_{\mathrm{f}}$ & $1.67 \times 10^{-2}$ & -4.09 & $12.7(0.1)$ \\
\hline
\end{tabular}

value of $\delta_{2}$ for the $n$-butyl ester when calculated from Eqn. 1.

Hagen (1979) reported values for the physicochemical properties of hydrocortisone and its solubilities in $n$-hexane, cyclohexane, carbon tetrachloride, toluene and benzene, given in Table 5 . Hydrocortisone provides an example of an organic compound with a high melting point, $212^{\circ} \mathrm{C}$. From this data, it can be shown that, using the assumption that $\Delta C_{\mathrm{p}}$ equals zero the value of $\ln a_{2}$ is -5.27 , while using the alternate assumption of the entropy of fusion gives $\ln a_{2}$ equal to -4.09 . The ideal solubility on a mole fraction basis, based on the former assumption, then, is over 3 times the solubility calculated using the latter assumption. Yet despite this extreme difference in ideal solubility, the "best-fit" $\delta_{2}$ values differ by only 0.3 hildebrand units.

The heat capacity assumption can play a significant role in the value of the ideal solubility only if the difference between the melting point and the solution temperature is large. Yet, in the extreme case of hydrocortisone, the solubility parameter is clearly insensitive to the heat capacity assumption. In practice, such a wide difference in temperatures will be the exception rather than the rule. The heat capacity assumption, then, should not have an appreciable effect on the value of the solubility parameter when it is calculated from solubilities in non-polar solvents using Eqn. 1. 


\section{Acknowledgements}

The authors would like to acknowledge the assistance of Steven Krawczyk and Eric Meade in the synthesis of the pentyl ester. S.H.N. was supported by an NIH Training Grant in Pharmacological Sciences, Grant GM 07767, H. Helfman Pharmacy Student Aid and Parke Davis/Warner Lambert.

\section{References}

Cave, G., Kothari, R., Puisieux, F., Martin, A.N. and Carstensen, J.T., Solubility parameters from maxima in solubility solvent plots. Int. J. Pharm., 5 (1980) 267-272.

Chertkoff, M.J. and Martin, A.N., The solubility of benzoic acid in mixed solvents. J. Am. Pharm. Assoc. Sci. Ed., 49 (1960) 444-447.

Flynn, G.L. and Yalkowsky, S.H., Correlation and prediction of mass transport across membranes I: Influence of alkyl chain length on flux-determining properties of barrier and diffusant. J. Pharm. Sci., 61 (1972) 838-852.

Funk, E.W. and Prausnitz, J.M., Thermodynamic properties of liquid mixtures: aromatic-saturated hydrocarbon systems. Ind. Eng. Chem., 62 (9), 8-15 (1970).

Hagen, T.A., Ph.D. Thesis, University of Michigan, Ann Arbor, MI, 1979.

Hagen, T.A. and Flynn, G.L., Solubility of hydrocortisone in organic and aqueous media: evidence for regular solution behavior in apolar solvents. J. Pharm. Sci., 72 (1983) $409-414$.

Hildebrand, J.H., Solubility. XII. Regular solutions. J. Am. Chem. Soc., 51 (1929) 66-80.
Hildebrand, J.H. and Scott, R.L., The Solubility of Non-Electrolytes, 3rd edn., Reinhold, New York, 1950.

Hildebrand, J.H., Prausnitz, J.M. and Scott, R.L., Regular and Related Solutions, Van Nostrand Reinhold, New York, 1970.

Hoy, K.L., New values of the solubility parameters from vapor pressure data. J. Paint Technol., 42 (1970) 76-118.

James, K.C. and Roberts, M., The solubilities of the lower testosterone esters. J. Pharm. Pharmacol., 20 (1968) 709-714.

Kertes, A.S., Solubility and activity of high-molecular amine hydrochlorides in organic solvents. J. Inorg. Nucl. Chem., 27 (1965) 209-217.

Khalil, S.A., Moustafa, M.A. and Abdullah, O.Y., The use of the solubility parameter as an index of drug activity. Can. J. Pharm. Sci., 11 (1976) 121-126.

Martin, A.N., Newburger, J. and Adjei, A., Extended Hildebrand solubility approach: solubility of theophylline in polar binary solvents. J. Pharm. Sci., 69 (1980) 487-491.

Martin, A.N. and Carstensen, J.T., Extended solubility approach: solubility parameters for crystalline solid compounds. J. Pharm. Sci., 70 (1981) 170-172.

Restaino, F.A. and Martin, A.N., Solubility of benzoic acid and related compounds in a series of $n$-alkonols. $J$. Pharm. Sci., 53 (1964) 636-639.

Scatchard, G., Equilibria in non-electrolyte solutions in relation to the vapor pressures and densities of the components. Chem. Rev., 8 (1931) 321-333.

Sunwoo, C. and Eisen, H., Solubility parameter of selected sulfonamides. J. Pharm. Sci., 60 (1971) 238-244.

Yalkowsky, S.H. and Zografi, G., Calculation of partial molal volumes in micellar systems. J. Pharm. Sci., 61 (1972) 793-795.

Yalkowsky, S.H., Flynn, G.L. and Slunick, T.G., Importance of chain length on physicochemical and crystalline properties of organic homologs. J. Pharm. Sci., 61 (1972) 852-857. 